\title{
Cusp Tethering Severity
}

National Cancer Institute

\section{Source}

National Cancer Institute. Cusp Tethering Severity. NCI Thesaurus. Code C127559.

The qualitative measurement of the severity of tethering of a cardiac valve cusp. 